\title{
The assessment of pain in advanced cancer
}

\author{
Robert G Twycross The Churchill Hospital, Headington, Oxford
}

This is one of a group of papers read at the London Medical Group conference on 'Pain: a necessity?', which was held in Charing Cross Hospital Medical School, London in February 1978. Dr Twycross argues that complete assessment implies the ability not only to make a diagnosis but also to initiate appropriate treatment. Describing the site, severity and quality of the pain is only the first step. A doctor needs to:

I) Be aware of the range of diagnostic possibilities

2) Appreciate the influences of non-physical factors such as mood and morale

3) Be aware of the range of treatment options

4) Establish realistic objectives with the patient

5) Reassess at appropriate intervals to review treatment, monitor side-effects, and develop and maintain a good working relationship with the patient.

Traditionally we are taught to assess pain by determining its PQRST characteristics (Table I). Unfortunately a blind belief in the efficacy of so simple an approach may hinder rather than help the doctor in the assessment of the patient's pain. Determination of the PQRST characteristics is only the beginning, providing a description of the pain but no more. Complete assessment implies the ability to make a diagnosis. This demands a grasp of both general and neurological anatomy, an understanding of the phenomenon of referred pain and a knowledge of the range of pathological processes which are potential causes of pain. From the history and clinical examination, supplemented if necessary by $x$-ray scan, or other test, it should be possible to develop a fairly clear mental picture of the physical mechanisms underlying the pain. Then, assessment completed and diagnosis made, treatment is initiated. We are familiar with this

\section{Table I The PQRST characteristics of pain ${ }^{1}$}

'Tell me about your pain.'

'Where is it?'

\begin{tabular}{ll}
\hline$P$ alliative factors & $\begin{array}{l}\text { 'What makes it less intense ?' } \\
\text { 'Wovocative }\end{array}$ \\
$Q$ uality & 'What makes it worse ?' \\
$R$ adiation & 'Does it spread anywhere else ?' \\
$S$ everity & 'How severe is it ?' \\
$T$ emporal factors & 'Is it there all the time, or does it \\
& come and go ?' \\
\hline
\end{tabular}

sequence of events in relation to, say, acute abdom $\stackrel{\widetilde{D}}{\Omega}$ inal pain, but often fail to apply the same relentless logic in assessing pain in other circumstances. $\vec{a}$

My own clinical involvement is largely confined to advanced cancer. Assessment needs to be asu thorough in this area as in any other. Cancer can cause pain in any part of the body and by a variet of mechanisms. Many patients have more than on pain. Moreover, alleviation of one pain may unmask another or a new pain may develop. To cope with what is often a complex situation, I find the use of 3 body chart to record pain data a great help (Fig. I)? Generally, patients put on a brave face for the doctor. Patients in severe pain do not always loo: distressed. Accordingly, intensity of pain is assesse $\Phi^{+}$ not only by the patient's description but also b $\overrightarrow{5}$ discovering what drugs have failed to relieve, whethe空 sleep is disturbed, and in what way activity is limited ("How long is it since you went out?" "ê" "What are you doing around the house?", In addition, the patient's spouse should be intre viewed. Often it is only the latter's comments thats give the true picture - though, when the pain is relieved, the patient frequently concurs spono taneously with the spouse's earlier opinion. $\mathbb{P}$ patient who is obviously in distress and who says $\overrightarrow{0 \overrightarrow{0}}$ implies that "It's all pain, doctor" is best thought of as having 'overwhelming' pain, that is, severe pain compounded by anxiety, depression and loss of morale.

\section{Diagnostic possibilities}

A diagnosis of cancer does not necessarily mean that the malignant process is the cause of the pain. peptic ulcer, cystitis or a musculo-skeletal disorde? may be responsible and are all conditions that benefit from more specific treatment. If the pain is. due to the cancer, it is important to determine the mechanism(s) of the pain(s) as treatment frequently varies according to mechanism.

Cancer pain may be caused by soft tissue infiltra N tion, bone involvement, nerve compression, elevate $\phi$ intra-cranial pressure, lymphoedema and viscera distension or obstruction. Infection in a malignan ulcer and muscle spasm secondary to bone involve? ment or deformity also occur. Constipation ando bedsores are common indirect forms of cancer pain? Asking the patient to point to the site of the pain an $\$$ to describe it will usually yield sufficient clues to make the correct diagnosis. 


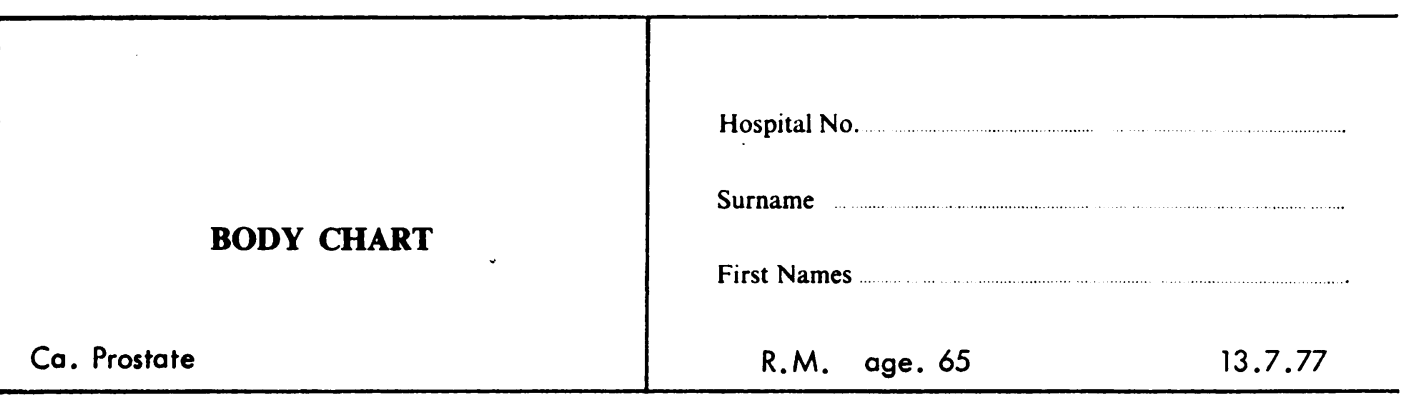

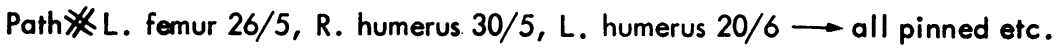

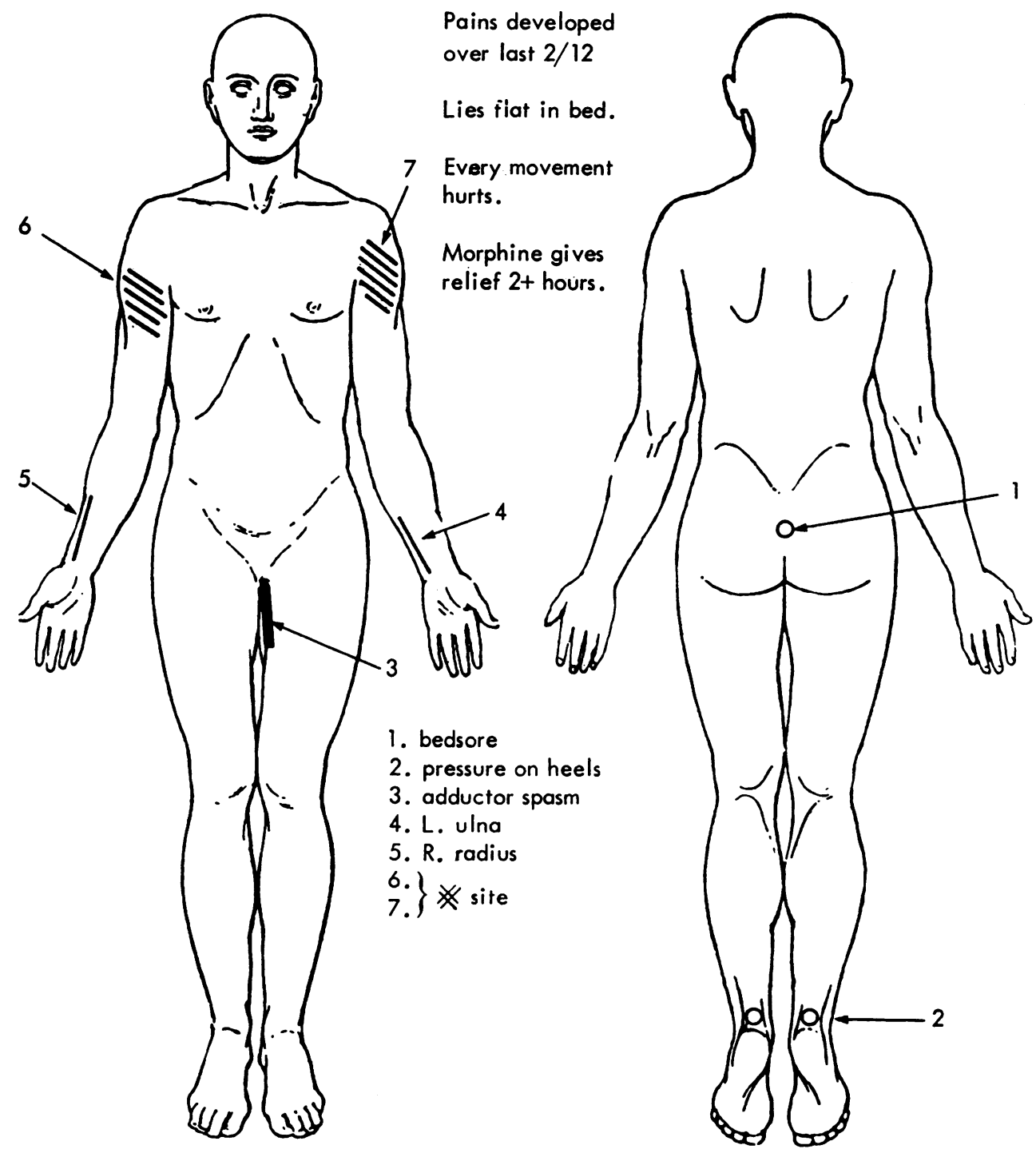

Note jumps when touched. Use analgesics + diazepam.

Fig I Body chart used to record pain data relating to 65-year-old man with cancer of the prostate gland. 
A four-year-old child with an inoperable pontine glioma experienced increasing pain in the head and occipital region. She lay flat all the time because elevation of the head caused a marked increase in pain. With this history it was necessary to postulate a local source of pain (possibly caused by post-radiation meningeal adhesions) in addition to the diffuse headache of secondary hydrocephlus (which would have been helped by a more erect posture). The diffuse pain was relieved by small regular doses of morphine but not until she was transferred from a King's Fund to an Ellison bed (which elevates head, neck and trunk in unison) was it possible for the child to sit up without pain. Subsequently it became possible to transfer the child from bed to a high-backed reclining chair and, eventually, to lift her onto her mother's lap. This suggested that some of the pain had been caused by spasm of the neck muscles, and that the confidence engendered by the ability to sit up in bed allowed additional manoeuvres to be undertaken without pain.

\section{Non-physical factors}

Pain is a dual phenomenon, one part being the perception of the sensation and the other the patient's psychological reaction to it. It follows that a person's pain threshold will vary according to mood and morale (Fig. 2). Attention must therefore be paid to factors that modulate pain threshold, such as anxiety, depression, and fatigue (Table II). Much can be done to alleviate pain by explaining the mechanism underlying the pain (this reduces

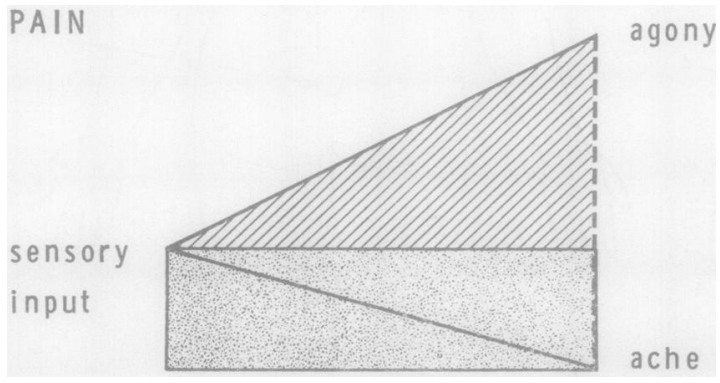

Fig 2 For any given noxious stimulus the pain experienced varies from ache to agony and depends on the psychological reaction of the sufferer to his discomfort.

Table II Factors affecting pain threshold

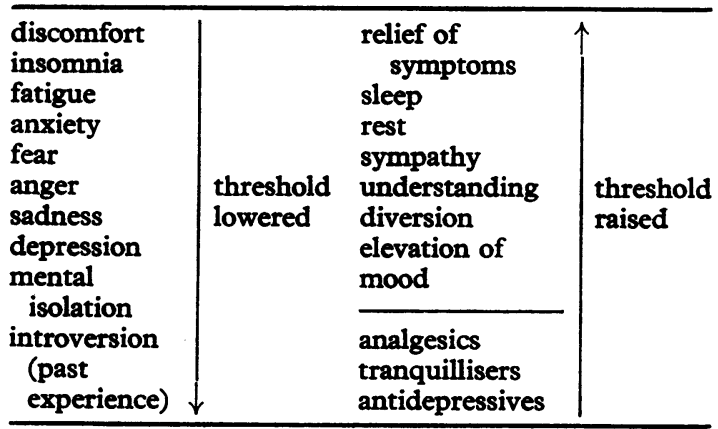

anxiety) and by a continuing concern for the patient? (this raises morale). Ignoring mental and social factors may result in otherwise relievable painm remaining intractable. At a weekly seminar for trainee practitioners ${ }^{2}$ discussion once centred on? the problem of a patient with metastases in bone from disseminated breast cancer whose pain was not relieved by narcotic analgesics. During the seminar? it was suggested that the pain was intractable as the woman was angry because her doctors anf relatives would not admit that she was dying nor discuss the problems this created. This proved to be the right explanation; a full and frank discussionresulted in a dramatic improvement in her mentaL state and she no longer complained of pain.

\section{Treatment options}

Relief of pain may be achieved by one or more the following methods:

I) Modification of the pathological process

2) Elevation of pain threshold

3) Interruption of pain pathways

4) Immobilisation

Even in far-advanced cancer, modification of the pathological process by means of radiation, chemo therapy, or hormone treatment should always bछ considered. As in other areas of oncology, itôis important to ensure that the treatment is not wose than the disease. If radiation or an oestrogenois prescribed, this does not mean that analgesics shout be withheld. The best results are obtained by adopting a 'broad-spectrum' approach, using two of more treatments in combination (Table III).

The use of analgesics and other drugs is best seer $\overrightarrow{\vec{D}}$ as but one way - generally a powerful way - of elevating the patient's pain threshold. Their use is fully discussed elsewhere. ${ }^{3.4}$ Interruption of pain. pathways refers to chemical neurolysis (nerves blocks) and neurosurgical techniques (e.g. rhizotomy spinothalamic tractotomy). Such procedures are of value in nerve compression/infiltration pain that is. inadequately relieved by the use of analgesics and of corticosteroid.

Some patients continue to experience pain on movement despite analgesics, other drugs, radio $\frac{D}{7}$ therapy and nerve-blocks. In these, the situation may be improved by suggesting commonsense modifications to daily activity. For example, a man may continue to struggle to stand while shaving unless the doctor suggests that sitting would be a good idea. Such a suggestion is accepted more readily if accompanied by a simple explanation of why weight-bearing precipitates or exacerbates the pain. Individually designed plaster or plastic supports for patients with multiple collapsed verte $\frac{T}{0}$ brae or Thomas splints for femoral pain are occa $\frac{\text { ते }}{\mathbb{N}}$ sionally necessary to overcome intolerable pain on? movement in bedfast patients. 
Table III Treatment of pain in advanced cancer

\begin{tabular}{lllll}
\hline Mechanism & $\begin{array}{l}\text { Analgesic and } \\
\text { adjuvants }\end{array}$ & Co-analgesic & $\begin{array}{l}\text { Non-drug } \\
\text { treatment }\end{array}$ & Other measures \\
\hline
\end{tabular}

I. Soft tissue infiltration

2. Bone involvement

3. Nerve compression

4. Raised cerebral pressure

5. Lymphodema

6. Abdominal visceral

a) epigastric

b) hypogastric

7. Ulceration - infection

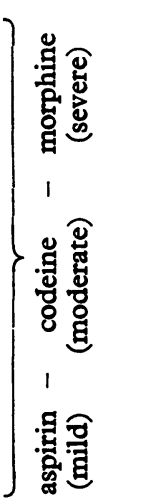

specific

treatment

$\left\{\begin{array}{ll}\text { aspirin } & \text { radiotherapy } \\ \text { prednisolone } & \text { nerve block } \\ \text { dexamethasone } & \\ \text { diuretic } & \text { compression sleeve } \\ & \\ & \text { coeliac axis } \\ & \text { ganglion block } \\ & \text { (presacral block)* }\end{array}\right\}$

8. Constipation

9. Second pathology

*The use of nerve blocks for lower abdominal pain is limited by probability of causing urinary retention.

Internal fixation or the insertion of a prosthesis should be considered if a pathological fracture of a long-bone occurs, as these measures obviate the need for prolonged bedrest and pain is usually relieved. The decision whether or not to treat surgically depends on the patient's general condition; but, whereas in bronchial carcinoma or malignant melanoma pathological fracture often presages death, in breast cancer this is not generally so, particularly if the tumour is hormone sensitive. The median survival after the first or only pathological fracture associated with breast cancer is about six months ranging from two months to four years. ${ }^{4}$

\section{Realistic objectives}

A recent survey of patients with persistent pain suggested that patients' expectations in relation to relief are lower than they need be. Although, in some, relief may be obtained fairly easily, it is important to bear in mind that with others, particularly those who have pain on movement and those whose pain is compounded by severe anxiety or depression, it may take three to four weeks of inpatient treatment to achieve satisfactory control. However, in all patients it should be possible to achieve some improvement within 24 to 48 hours. Although the ultimate aim is always complete freedom from pain, a doctor will be less disappointed but, paradoxically, more successful if he aims at 'graded relief'. Moreover, as some pains respond more readily than others, improvement should be assessed in relation to each pain.

The initial target should be a pain-free, sleep-full night. Many patients have not had a good night's rest for weeks or months. To sleep through the night and wake refreshed is a boost to both the doctor's and the patient's morale. Next, one aims for relief at rest in bed or chair during the day finally, for freedom from pain on movement. The former is always eventually possible; the latter iso not. However, the encouragement that relief at night and when resting during the day brings gives the patient new hope and incentive and enables him to begin to live again despite limited mobility. Freed from the day and nightmare of constant pain, his last weeks or months take on a new look.

\section{Reassessment}

With cancer one is dealing with a progressive pathological process. This means that new pains may develop or old pains re-emerge. It should not be assumed that a fresh complaint of pain merely calls for an increase in a previously satisfactory analgesic regimen; it demands reassessment, an explanation to the patient and, only then, modification of drug therapy or other intervention. The probability of the initial prescription being inadequate increases with the intensity of pain. Patients should, therefore, be reassessed within hours if the pain is overwhelming, or after one or two days if severe or moderate. If troublesome or unacceptable sideeffects result, treatment may need to be modified. In addition, the relief of the major pain may allow a second less severe pain to become apparent.

An 85-year-old man with carcinoma of the prostate and pain in the right femur caused by a metastasis was treated with aspirin and morphine. Casual questioning 
the next day indicated that, although less severe, he was still in pain. Further questioning revealed that the site of pain was now retrosternal and epigastric; he had no femoral pain at all. The dose of morphine was left unaltered, and the prescription of an antacid resulted in complete relief.

In the case of the man with overwhelming pain (Fig. I), it was necessary to review progress in relation to each pain. On the second day he was reluctant to admit that several of the pains were less intense but, judging by his reactions to passive movement, they undoubtedly were. Possibly his reluctance was due to a shifting base-line of reference. It was necessary to remind him of the difference I observed and to encourage him to accept that the pains were beginning to ease. It was a case of 'chipping away' at his total pain experience until eventually, after a week or so, he was considerably more comfortable and able to sit up in bed.

\section{Acknowledgements}

Tables II and III and Fig. I are reproduced by permission of Pitman Medical, Tunbridge Wells, England.

\section{References}

${ }^{1}$ Gray, $J$ (1977). A pain in the neck - and shoulder. Pain Topics, 1, No. 3, 6.

2Anonymous, (1971). Vocational training for general practice. British Medical fournal, 2, 704-706.

${ }^{3}$ Vere, D W (1978). Pharmacology of morphine drugs used in terminal care. In: Topics in Therapeutics 4, edited by $D W$ Vere, pp. 75-83. Pitman Medical, Tunbridge Wells.

'Twycross, R G (1978). Bone Pain in Advanced Cancer, ibid., pp. 94-110.

${ }^{5}$ Twycross, $R$ G (1977). Care of the terminal patient, In: Breast Cancer Management - early and late. Edited by B A Stoll, pp. 157-163. Heinnemann Medical Books Ltd, London.

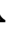

